\title{
De novo Glomerular Disease and the Significance of Electron Microscopy in Renal Transplantation
}

\author{
Surya V. Seshan Steven P. Salvatore \\ Department of Pathology and Laboratory Medicine, Weill Cornell Medicine, New York, NY, USA
}

\begin{abstract}
Keywords
Renal transplantation - De novo diseases - Electron microscopy · Focal segmental glomerulosclerosis . Membranous glomerulonephritis · Collapsing glomerulopathy $\cdot \lg$ A nephropathy $\cdot$ Pediatric donor glomerular diseases
\end{abstract}

\begin{abstract}
Background: De novo glomerular diseases comprising those both common and unique to transplant may develop in the renal allograft leading to posttransplant proteinuria, hematuria, or allograft failure. Electron microscopy (EM) is a useful adjunct to the standard light and immunofluorescence microscopy for accurately diagnosing these diseases and subsequently aiding the clinician in initiating appropriate treatments. Summary: De novo diseases are those new-onset diseases in renal transplantation that are unrelated to the original kidney disease in the recipient. They include virtually any primary or secondary glomerular, tubulointerstitial, or vascular diseases, ranging from subclinical to clinically overt, having acute, subacute, or chronic clinical presentations. This review focuses on common or significant, mainly glomerular, entities, with particular attention to the EM findings. The time of onset, stage, and severity of these diseases may often be modified by the current immunosuppressive protocols and other donor and recipient predisposing char-
\end{abstract}

karger@karger.com www.karger.com/gdz

Karger $\stackrel{\text { ' }}{5}$

GOPEN ACCESS
(C) 2021 The Author(s)

Published by S. Karger AG, Basel

This is an Open Access article licensed under the Creative Commons Attribution-NonCommercial-4.0 International License (CC BY-NC) (http://www.karger.com/Services/OpenAccessLicense), applicable to the online version of the article only. Usage and distribution for commercial purposes requires written permission. acteristics. Key Messages: A renal allograft biopsy not only improves our understanding of the pathophysiology but also provides diagnostic accuracy prognostic information, and potential for reversibility. In some cases, the biopsy leads to detection of unsuspected or clinically asymptomatic de novo diseases in the setting of other concomitant rejection processes, infection, or toxicity, which can dictate appropriate therapy. Routine EM in transplant kidney biopsies is a valuable modality in recognizing fully developed or early/subtle features of evolving de novo diseases, often during the subclinical phases, in "for cause" or surveillance/ protocol allograft biopsies.

(c) 2021 The Author(s)

Published by S. Karger AG, Basel

\section{Introduction}

De novo/new-onset disease in a renal transplant is defined as the occurrence of a newly acquired nonrejectionrelated pathologic process affecting the allograft, which differs from the recipient's original kidney disease which led to failure of the native kidneys. Hypothetically, almost the entire spectrum of glomerular, tubulointerstitial, and vascular lesions can occur with few exceptions as de novo (new onset) diseases, unrelated to the original kidney disease, during the life of a renal allograft, having a substantial impact toward graft survival $[1,2]$. Electron micros- 
Table 1. De novo renal diseases causing renal transplant dysfunction

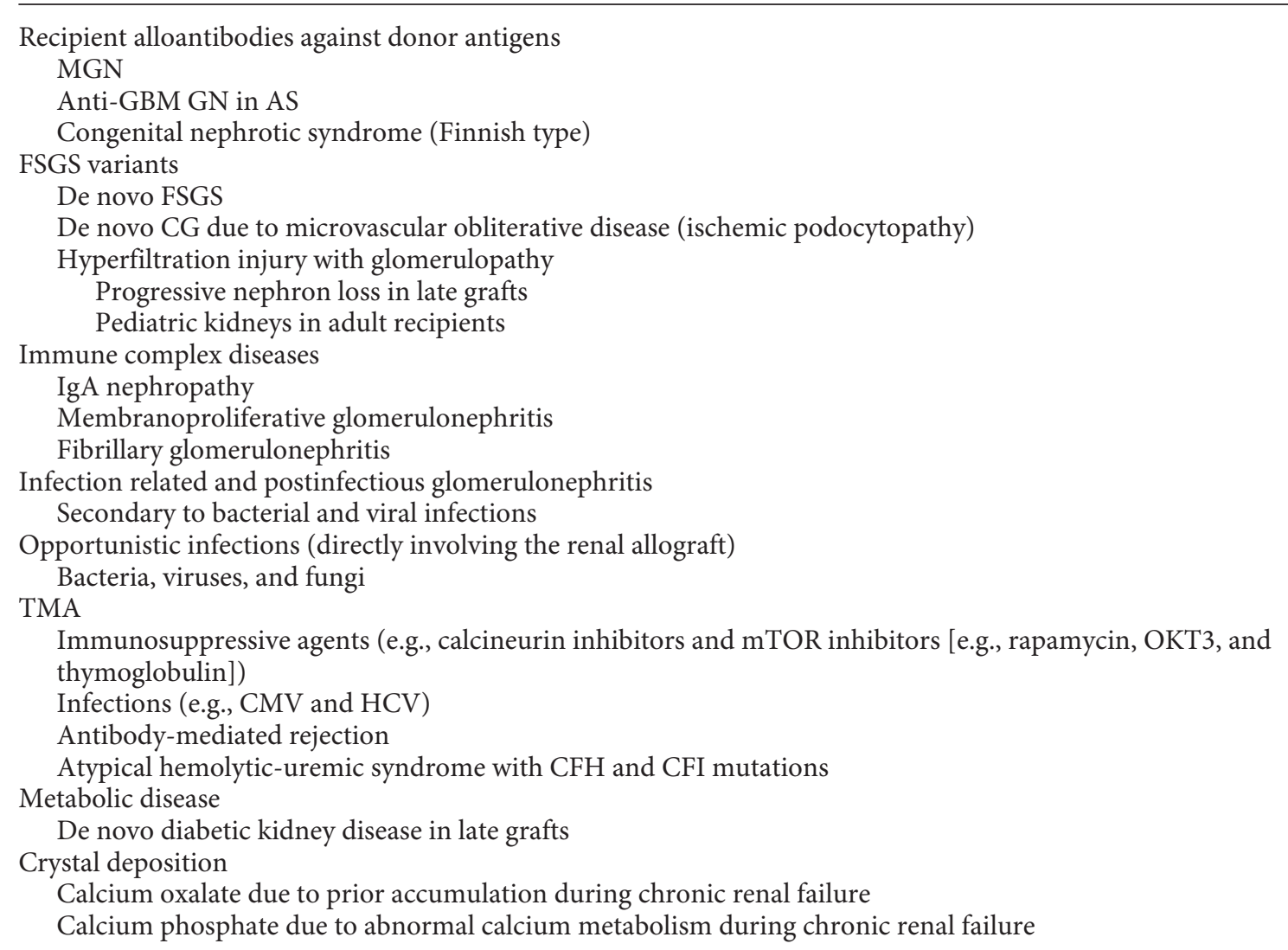

MGN, membranous glomerulonephritis; GBM GN, glomerular basement membrane-mediated glomerulonephritis; AS, Alport syndrome; FSGS, focal segmental glomerulosclerosis; CMV, cytomegalovirus; HCV, hepatitis C virus; CG, collapsing glomerulopathy; TMA, thrombotic microangiopathy.

copy (EM) is best utilized in the diagnosis of glomerular diseases. However, a group of common or special de novo glomerular diseases (those associated with transplant-related alloantibodies) mainly in the setting of transplantation that deserve attention are listed in Table 1, modified from reference [3]. They can also superimpose on other preexisting allograft pathologic findings. The time of onset, stage, and severity of these diseases may often be modified by the current immunosuppressive protocols and other donor and recipient predisposing characteristics.

The exact prevalence of de novo glomerular diseases is unknown, but 1 study suggests an incidence of up to $10 \%$ at 15 years [2]. Together with recurrent diseases, de novo glomerular diseases form the third leading cause of renal allograft dysfunction and failure. However, no definite pattern or frequency of occurrence of a specific form of de novo glomerular disease is identified and the clinical features depend on the type of lesion affecting the al- lograft. A minimal or modest degree of posttransplant proteinuria is not unexpected in the normal setting, in acute rejection process or chronic allograft failure. However, higher levels of proteinuria that are close to or over the nephrotic range $(2.5$ to $>5 \mathrm{~g} / 24 \mathrm{~h})$ indicate the presence of a glomerular disease regardless of etiology [4]. They may often be associated with recurrent/de novo glomerular lesions or early or late rejection associated glomerulopathy requiring EM for diagnosis and differentiation from the former lesions [5]. Usual clinical renal disease workup for proteinuria, hematuria, serum chemistries that include the basic metabolic panel, appropriate serological testing where indicated for infections, and autoimmune and monoclonal protein-associated diseases are suggested.

Our center does EM on all transplant kidney biopsies, but this is not a universal practice. For a definitive diagnosis and any subtle findings, aiding in predicting treat- 

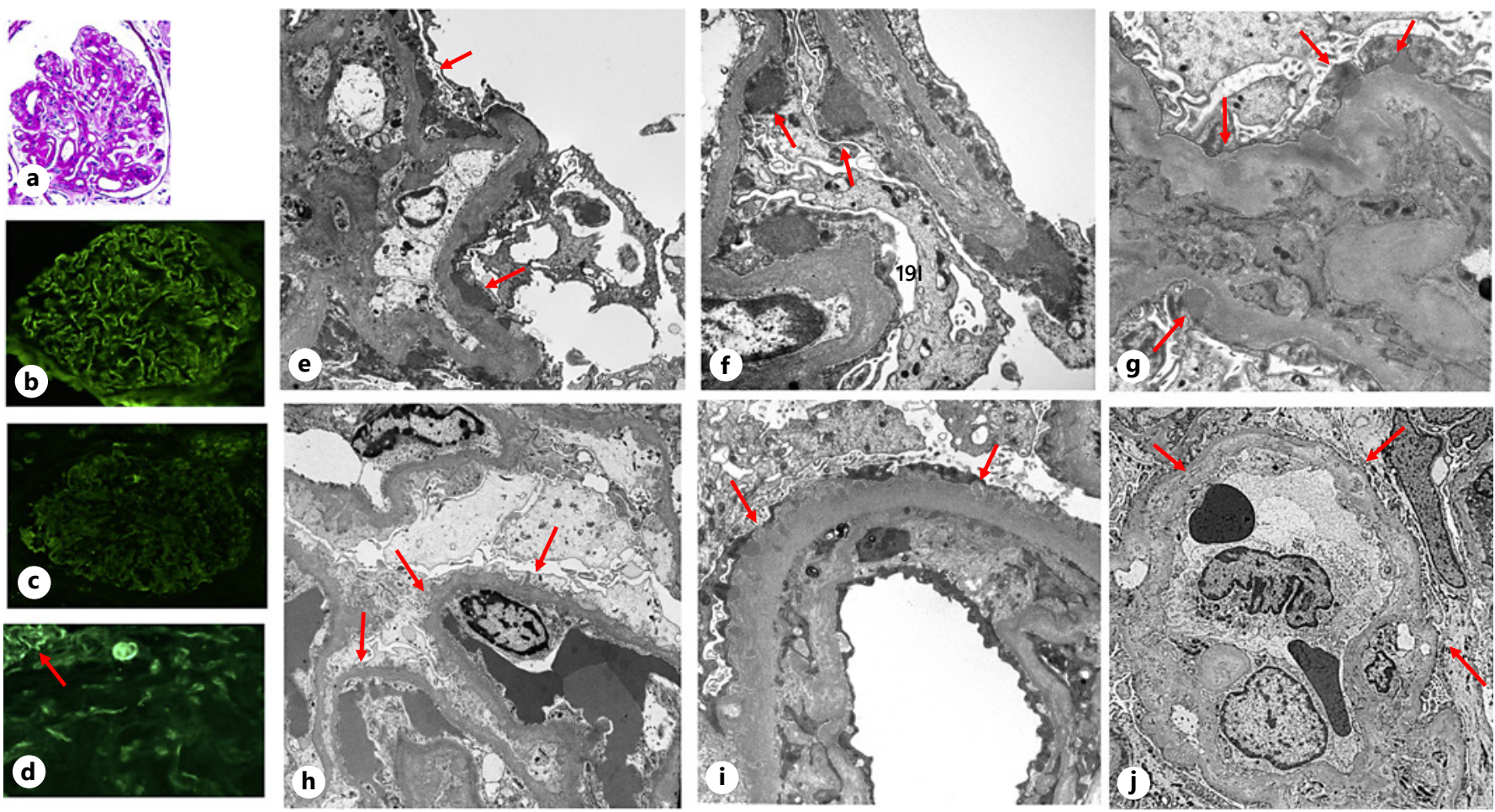

Fig. 1. De novo MGN in a patient with nephrotic range proteinuria after 6 years following renal transplantation. a Glomerulus showing mild mesangial and peripheral capillary wall thickening (PAS $\times 400$ ). b Global, finely granular-positive staining for polyclonal IgG along the glomerular capillary walls $(\operatorname{IgG} \times 400)$. c IF staining on paraffin-embedded tissue for PLA2R is negative within the glomerular deposits (PLA2R $\times 400)$. d Positive C4d staining along peritubular capillaries and in the glomerular capillaries with a granular texture, a portion seen in the upper left corner (arrow) (C4d $\times 400)$. e-g EM: The glomerular deposits can be small or sparse and often irregular in size and shape, some assuming domeshaped deposits lacking basement membrane spikes, along with widespread foot process effacement in the vicinity of the deposits. The lamina densa may be variably thickened $(\times 6,000(\mathbf{e}), \times 15,000$ $(\mathbf{f}), \times 150,000(\mathbf{g}))$. $\mathbf{h}$ Long-standing cases may show basement membrane spikes and resolution of the deposits giving rise to irregular basement membrane remodeling with persistent foot pro- cess effacement (arrows) $(\times 6,000)$. i A portion of a glomerular capillary wall showing small subepithelial and intramembranous electron dense deposits, foot process effacement, and thickening of the lamina densa. Note subendothelial widening with cellular interposition and new basement membrane formation with organization of the lucent space, without immune complex deposits. These suggest chronic endothelial injury and transplant glomerulopathy, most probably associated with antibody-mediated rejection $(\times 10,000)$. j Glomerular capillary loop from another patient showing attenuation of the lamina densa with organization of the subendothelial space, segmental cellular interposition, circumferential new basement membrane material formation, and margination of inflammatory cells suggesting transplant glomerulopathy with rare small or no evidence of basement membrane deposits in a patient who was previously diagnosed with an early de novo MGN (×6,000). MGN, membranous glomerulopathy; IF, immunofluorescence; EM, electron microscopy. ment response or outcome [6], EM is recommended in renal allograft biopsies presenting with nephrotic range proteinuria, selected cases of subnephrotic proteinuria, some cases of hematuria or with certain findings indicating a glomerular disease process by light microscopy and immunofluorescence (IF). Therefore, if not performing routine EM, most laboratories should reserve a small sample of cortical tissue for EM at the time of triaging for potential future studies. It should be noted that only de novo associated glomerular diseases are covered in this review. Rejection associated glomerular lesions including transplant glomerulopathy are covered elsewhere in this special issue of the journal.

\section{Pathologic Features}

A complete examination by light microscopy of the renal biopsy, as performed for a native kidney disease, with adequate levels and routine special stains, is essential. In addition, the tissue should be subjected to the usual panel of IF staining (including C4d) and EM, where feasible, or 
in clinically suspected cases. Several laboratories around the world have opted to perform routine IF panels used in native kidney disease along with $\mathrm{C} 4 \mathrm{~d}$ staining in order to obtain information on early detection of asymptomatic recurrent/de novo disease in the allograft. On occasion, early/mild form or established/known LM, IF, and/or EM features of the original disease could also be identified. Concomitant features of rejection may be present.

The role of EM in this setting is crucial in the identification of early or a subtle lesion in the asymptomatic stage, prompting appropriate changes in management. Apart from clinical findings, suspicious glomerular lesions in transplant renal biopsies that can trigger further IF or EM testing include intraglomerular cell proliferation, inflammatory cell infiltration, and capillary wall changes of irregularity, thickening, or additional contours.

\section{Selected De Novo Glomerular Diseases Are Described below, Some of Which Require EM for Diagnosis or May Be Confirmatory}

\section{De novo Membranous Glomerulopathy \\ Definition}

De novo membranous glomerulopathy (MGN) is a relatively uncommon form of posttransplant glomerular lesion occurring in about $0.3-2.1 \%$ of adult transplant cases, where the original end-stage kidney disease (ESKD) is not primary MGN, although some studies have shown a higher prevalence in pediatric renal transplant recipients. It has been encountered as early as 1 month posttransplantation, but with an interval that may range from 2 to several years. While the exact pathogenetic mechanism underlying de novo MGN has not been elucidated, the association of chronic antibody-mediated rejection, positive peritubular capillary C4d staining, and donorspecific antibodies is noted, particularly against HLA-DQ in many of these cases. The absence of PLA2R staining within the glomerular deposits suggests a possible link to an alloimmune response to an unknown tissue antigen [7-9]. There is a tendency for increased risk of de novo MGN in HCV-positive patients, contributing toward lower graft survival [10]. Although the terms "primary and secondary MGN" may in time be replaced by the respective putative antigens with a clinical setting, for example, PLAR2R, THSD7A, Nell-1, exostosin 1/exostosin 2 , and semaphorin $3 \mathrm{~B}$; these forms of MGN may also rarely occur as de novo lesions with corresponding circulating autoantibodies [11-13].

De novo Diseases in Renal

Transplantation

\section{Clinical Features}

De novo MGN presents with asymptomatic subnephrotic or nephrotic range proteinuria with stable graft function initially. Often, significant graft dysfunction is noted in the presence of a concomitant rejection process and/or other active or chronic graft parenchymal changes. Appropriate serologic studies could be indicated when infections or autoimmune diseases are suspected.

\section{Light Microscopy}

As in any form of developing or early MGN, the glomerular capillary wall changes may be minimal and not apparent by LM, but some degree of mesangial thickening and hypercellularity may be noted in about one-third of the cases (Fig. 1a). These cases are often initially discovered only when routine IF and EM are performed on transplant kidney biopsies. A small proportion of cases may disclose evidence of antibody mediated rejection or early-transplant glomerulopathy [5].

\section{IF Microscopy}

Finely granular glomerular capillary wall staining for deposits of mainly polyclonal IgG and C3 is seen (Fig. 1b). In cases of concurrent antibody-mediated rejection, there is positive linear $\mathrm{C} 4 \mathrm{~d}$ staining in the peritubular capillaries and finely granular staining within the glomerular deposits (Fig. 1d). This differs from primary MGN by the lack of reactivity to PLA2R antibodies (Fig. 1c) [9]. However, other potential forms of de novo MGN (PLA2R, THSD7A, NELL-1, exostosin 1/exostosin 2, semaphorin $3 \mathrm{~B}$, etc.) could also be considered using specific antibodies that can help classify the underlying etiology [11-13]. Significant heterogeneity of staining for IgG subsets exists within these forms of MGN $[12,13]$, where majority of PLA2R-positive and TSHD-positive cases display IgG4, nearly $20 \%$ of these cases may be negative. Additionally, variable staining for IgG1 and IgG2 may also be observed along with some IgG4 in a proportion of cases showing other newly described antigens in MGN [11-13]. The composition of the deposits may appropriately vary with infectious or autoimmune etiologies.

\section{Electron Microscopy}

In the initial stages with only proteinuria, the glomerular capillary basement membrane deposits can be extremely small and sparse, sometimes termed "stage 0 ," with IF staining driving the diagnosis. While this type of staging has been described in early recurrent MGN [14], similar findings may be detected in early de novo MGN lesions as well, particularly in protocol biopsies. In such

Glomerular Dis 2021;1:160-172 


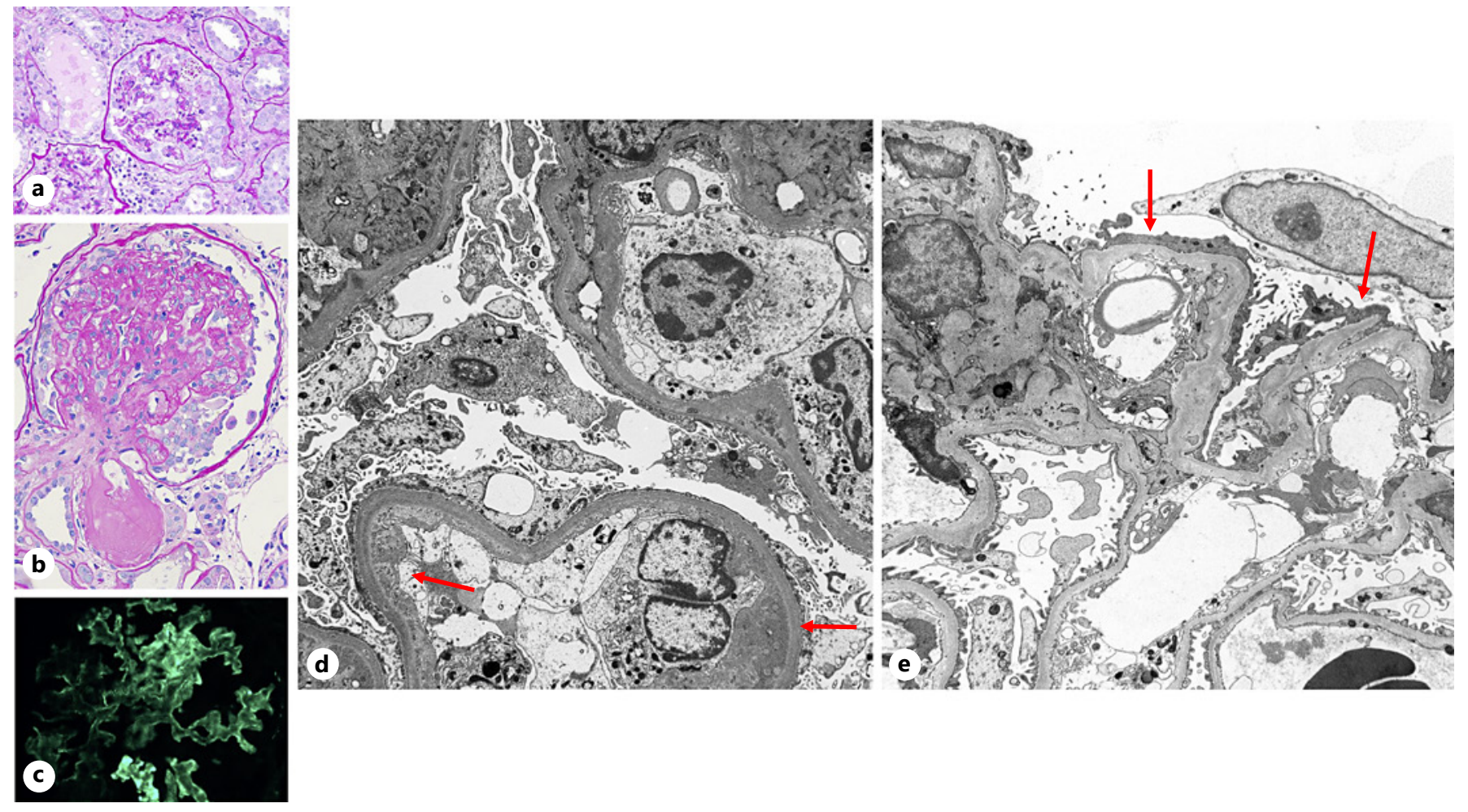

Fig. 2. De novo CG. a Two glomeruli showing global wrinkling and collapse with almost total occlusion of the capillaries surrounded by hyperplastic and vacuolated epithelial cells in the Bowman's space, some containing prominent protein droplets within the cytoplasm, while the other glomerulus appears normal with adjacent dilatation of the tubules with proteinaceous cast (PAS, $\times 400)$. b A glomerulus from a different case shows global capillary tuft collapse covered by hyperplastic epithelial cells and a hilar arteriole almost occluded by a large intimal and focal medial hyalin deposit (secondary to chronic calcineurin inhibitor toxicity), causing glomerular hypoperfusion and ischemic podocytopathy, now showing collapsing features (PAS $\times 400)$. c Nonspecific, granular IgM staining is noted in glomerular capillaries $(\operatorname{IgM} \times 400)$. d EM of a noncollapsed glomerulus from these cases may disclose variable distortion or foot process effacement, margination by inflammatory cells and endothelial swelling with segmental subendothelial accumulation of proteinaceous material suggesting an ongoing rejection process $(\times 6,000)$. e Other nonspecific glomerular changes may show normal thickness of basement membranes with focal foot process effacement, since severe podocyte changes are restricted to the affected collapsed glomeruli $(\times 6,000)$. EM, electron microscopy; CG, collapsing glomerulopathy. cases, there is diffuse foot process effacement with microvillus transformation, suggesting poorly visible small subepithelial deposits $(1 \mathrm{E}, \mathrm{F})$. In other cases, there are scattered, irregular subepithelial deposits, some of which may assume dome shape without or with early basement membrane spike formation (Fig. 1e-g), as seen in the classic stage 1 and stage 2 MGN, described by Ehrenreich and Churg [15]. With increased duration, these electrondense deposits form intramembranous deposits in stage 3 (Ehrenreich \& Churg) that may eventually show evidence of resolution, basement membrane remodeling and persistent foot process effacement, or partial recovery (stage IV Ehrenreich \& Churg [15] (Fig. 1h, i)). Mesangial deposits are not generally a feature in this setting, unless the underlying etiology of the de novo MGN is of infectious or autoimmune origin. In more chronic stages and particularly in cases with concomitant antibody-mediated rejection, glomerular endothelial injury, swelling, loss of fenestrations, and progressive glomerular basement membrane thickening with duplication and cellular interposition have been observed in previous studies with repeated biopsies (Fig. 2i, j). This may contribute toward enhanced or nephrotic-range proteinuria and lead to allograft dysfunction and failure [7, 9].

\section{De novo Focal Segmental Glomerulosclerosis \\ Definition and Background}

De novo FSGS is often a long-term morphological consequence in renal transplants, secondary to any form of glomerular injury, commonly secondary to medica- 
tions (calcineurin inhibitor toxicity or mTOR inhibitor effect, e.g., rapamycin), hypertension, and diabetic kidney disease with advancing microvascular sclerosis, infections, or rejection. This needs to be differentiated from recurrent FSGS, where the original ESKD was due to a variant of FSGS. Hyperfiltration-mediated visceral epithelial injury and FSGS can occur in transplants due to structural adaptation, following routine compensatory hypertrophy after a year or more in a functional allograft, reduced renal mass in long-standing transplants, advanced/progressive vascular sclerosis, or pediatric donor kidneys transplanted to adult recipients, as a consequence of size mismatch. Pathologic findings of de novo FSGS may resemble FSGS NOS type, collapsing glomerulopathy (CG) or perihilar type with other features of visceral epithelial cell hyperplasia and capsular adhesions with or without segmental hyalinosis. This can be accompanied by progressive tubulointerstitial scarring and vascular sclerosis. It is not entirely clear if EM study of the glomerulus to assess the presence or extent of foot process effacement or podocyte morphology is sufficient to separate the primary entity from the secondary forms in an allograft kidney biopsy in every case because of the ongoing immunomodulation by antirejection medications and other concomitant insults. Additional clinical information, along with those pertaining to donor status, immunosuppressive protocols, etc., may help determine the underlying pathophysiologic process.

\section{De novo CG}

Definition and Clinical Features

De novo CGs manifesting similar glomerular morphology as the idiopathic form in the native kidney or in recurrent CG may develop in genetically susceptible populations, presence of donor APOL1 high-risk genotypes, particularly from donors of African descent [16] or severe visceral epithelial injury due to obliterative microvascular disease, causing acute or subacute occlusion [17]. The development of this lesion contributes to poor prognosis (up to $50 \%$ graft loss in 1 year). Viral infections (e.g., HIV, parvovirus, $\mathrm{CMV}$, and $\mathrm{HCV}$ ) have also been implicated in its pathogenesis, prompting full serologic testing in suspected cases. The common clinical features in all these scenarios are variable to nephrotic range proteinuria which is often intractable to therapy, increased creatinine levels, and relatively rapid progression to ESKD. Most of the cases, regardless of underlying pathogenetic mechanisms, follow this course leading to allograft failure within 1-3 years posttransplantation. The category related with microvascular occlusive disease is associated with several disease processes that are capable of developing obliterative disease, such as chronic calcineurin inhibitor vascular toxicity causing medial and intimal hyalinosis, preexisting donor or recurrent diabetic kidney disease, progressive hypertension, thrombotic microangiopathy (TMA), atheroembolism, or vascular rejection of cell-mediated or antibody-mediated rejection processes.

\section{Renal Pathology}

The pathologic diagnosis is essentially made by LM showing segmental or mostly global wrinkling and collapse of the glomerular capillary tufts, in a focal or rarely diffuse distribution, with hyperplastic and vacuolated epithelial cells, occupying the Bowman's space, some containing PAS-positive protein resorption droplets, mostly capping the collapsed areas (Fig. 2a, b). The hilar arteriole is mostly occluded by extensive intimal and medial hyalinosis. The more chronic lesions may show segmental or global sclerosing changes. Focal tubular microcystic changes, interstitial inflammation, tubular atrophy, and interstitial fibrosis are also seen, depending on the duration of the lesions and posttransplant interval. The glomerular findings of de novo CG may be zonal, following the distribution of obliterative arteriopathy in some cases [17], suggesting ischemic injury of visceral epithelial cells or "ischemic podocytopathy" as the pathogenetic mechanism in those cases. No immune deposits (immunoglobulins or complement components) are identified by IF except for focal IgM staining in the areas of collapse or sclerosing changes (Fig. 2c). While the affected collapsed glomeruli display total foot process effacement with degenerative changes of the endothelial and mesangial cells and typical marked reactive visceral epithelial changes by EM, only nonspecific or nonsignificant features are noted in the other glomeruli, with focal or no substantial foot process effacement (Fig. 2d, e).

\section{Size Mismatch (Pediatric Donor Related) Glomerulopathy Secondary to De Novo Hyperfiltration Injury}

\section{Definition and Background}

The development of "size mismatch glomerulopathy (SMG)" which affects $<10 \%$ of adult recipients, is defined by the process of hyperfiltration injury and adaptation secondary to higher recipient blood pressure and blood flow within the glomeruli in pediatric donor allografts of $<3$ years of age [18]. Since the glomerular size in the pediatric donors is smaller and often immature, 

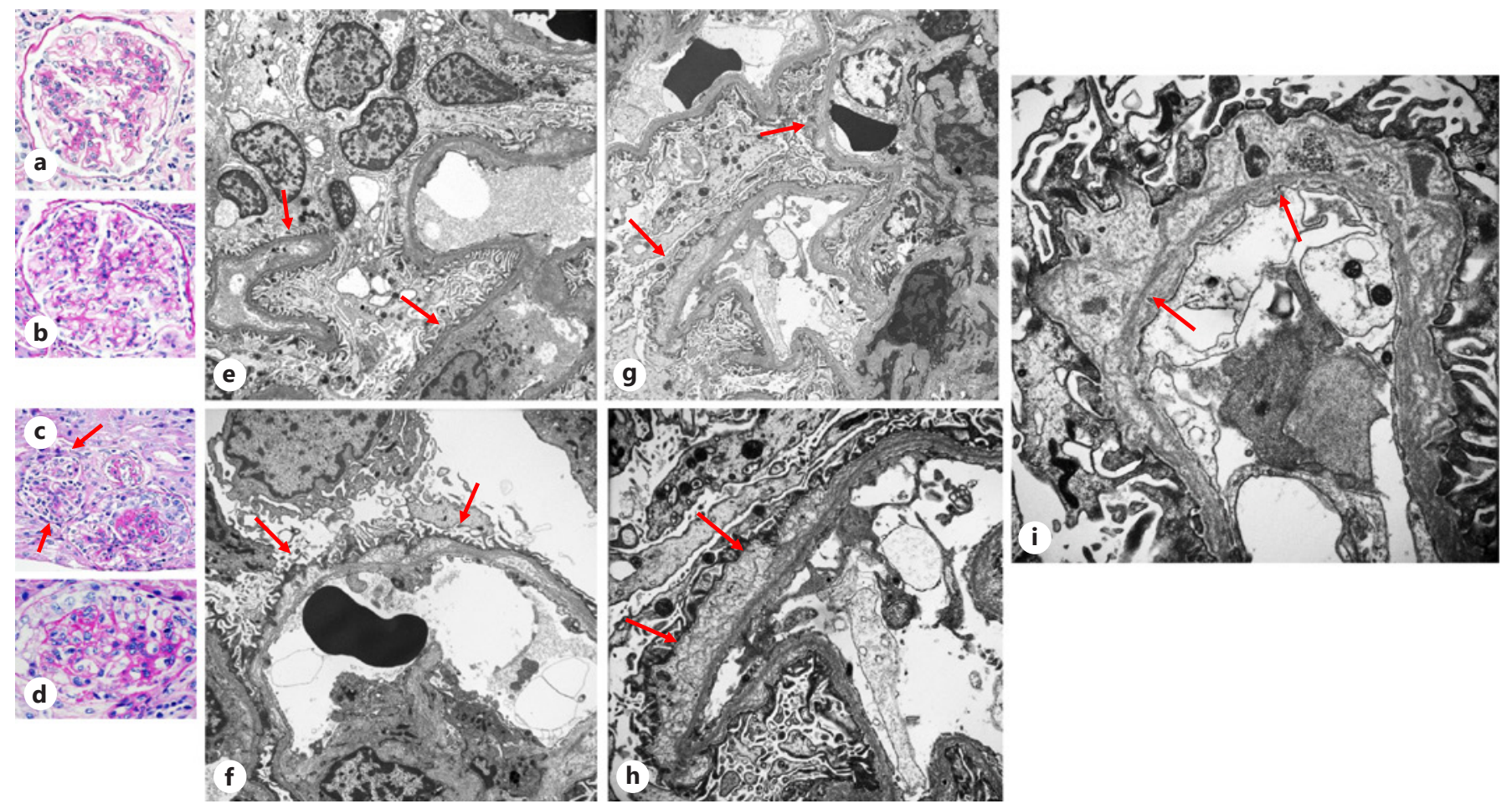

Fig. 3. Pediatric donor (hyperfiltration) glomerulopathy. The images in this figure are taken from 2 different patient transplant kidney biopsies with pediatric donors who presented with subnephrotic and nephrotic range proteinuria. a-d The light microscopic glomerular features can range from mild glomerular hypertrophy to minimal or moderate mesangial hypercellularity with or without segmental sclerosing or collapsing lesions showing hyperplastic epithelial cells on the surface. While the larger glomeruli represents an adaptation due to hyperfiltration post pediatric kidney transplantation to adult recipients, occasionally normal fetal or infantile glomeruli can also be visualized in (c) (arrows) (PAS $\times 400$ ). e-i The EM images of the glomerular capillaries show a range of capillary basement membrane changes which appear originally thin but disclose progressive segmental and focal irregular subepithelial expansion involving the lamina rara externa, with irregular lamellation of basement membrane material (arrows) having generally intact lamina densa and relatively preserved foot processes with focal distortion. Hyperplastic and vacuolated epithelial cells may also accompany these lesions as part of a podocytopathy as a result of hyperfiltration injury $(\times 6,000(\mathbf{e}), \times 8,000(\mathbf{f})$, $\times 6,000(\mathbf{g}), \times 12,000(\mathbf{h}), \times 20,000(\mathbf{i}))$. EM, electron microscopy; IF, immunofluorescence. with extremely thin glomerular capillary basement membranes, exposure to the direct mechanical adultlevel hemodynamic forces appears to stress and injure the glomerular epithelial cells, resulting in this unique lesion [19].

\section{Clinical Features}

Clinical renal disease may develop as early as 4 months to under 2 years, manifesting onset of variable subnephrotic and rarely nephrotic range proteinuria, with persistent microhematuria and hypertension in a few cases. While a smaller series of case studies have reported renal failure in less than a year, overall the creatinine levels are relatively stable or normal, in the long term. At 5 years posttransplantation, there is $>50 \%$ graft survival. A recent study showed that a higher donor age, donor weight, or donor kidney volume may be protective and may not tend to develop SMG in the recipient $[18,19]$.

\section{Light and IF Microscopy}

The light microscopic findings in SMG are characterized by variable enlargement and compensatory hypertrophy of the glomeruli following transplantation, mild to moderate mesangial hypercellularity (Fig. 3a, b), and focal segmental, and global glomerulosclerosis. They may also show evidence of segmental or global CG features, with hyperplastic and vacuolated epithelial cells filling the Bowman's space and covering the areas of collapse (Fig. 3c, d). Mild hypertrophy of the renal tubules is also observed, sometimes with increased epithelial protein resorption droplets suggesting a proteinuric state. On occasion, other transplant-related complications, such as re- 
jection process, polyoma virus-associated nephropathy, or even recurrent glomerular diseases are concurrently present. No specific IF staining is localized within the glomeruli in SMG, except for focal nonspecific trapping of IgM and/or C3.

\section{Electron Microscopy}

The glomerular basement membranes are markedly thin depending on the age of the pediatric donor kidney, with only focal foot process effacement. In addition, focal segmental irregular thickening and expansion of the basement membranes with rarefaction of subepithelial areas or lamina rara externa is noted. There is formation of new layers of the basement membrane material, having a focally interrupted lamellated appearance, mainly restricted to the zones of lamina rara externa (Fig. 3e-i). The lamina densa in these areas is generally intact with focal attenuation. The foot processes are mostly preserved with focal effacement or distortion in areas of basement membrane expansion. The overlying visceral epithelial cells are prominent and increased in number with variable swelling. The main differential diagnosis is recurrent focal segmental glomerulosclerosis with subepithelial irregularities and changes. Although the multi-lamellation of the basement membranes resembles Alport syndrome (AS) (which does not develop in a renal allograft), the lamina densa within the glomerular basement membranes is almost always affected along with significant foot process effacement in AS.

Similarly, renal functional and structural adaptations have been observed when an adult kidney is transplanted in pediatric patients with a smaller body size, creating another form of size mismatch glomerular appearance [20, 21]. The functional adjustment with regard to glomerular filtration rate to the body surface area is known to happen within 3 months after transplant [20]. However, in one study, morphologic changes in terms of posttransplant reduction in kidney volumes and size of the glomeruli were also documented at the end of 1 year [21]. These changes have been shown partly as a consequence of pretransplant abdominal cavity and posttransplant weight gain and somatic growth [21].

\section{De novo IgA Nephropathy}

Definition

De novo IgA nephropathy is defined as new-onset, non-donor-related IgA-dominant mesangial deposits in the allograft in patients who did not have IgA nephropathy in their native kidney. The finding of de novo IgA is relatively uncommon $[22,23]$ and have to be differentiated from latent or incidental IgA glomerular deposits in donor kidneys [24]. It is much more likely that IgA nephropathy in the allograft is due to recurrent or donor-related IgA nephropathy, even when the original cause of ESRD is unknown, or rarely IgA-associated infection-related GN [25]. Alternatively, IgA vasculitis (Henoch Schonlein Purpura Nephritis) with concomitant skin involvement may develop in the allograft recipient. Donor-related disease is not infrequent, depending on ethnicity of the donor and related versus nonrelated living donors. In one study, mesangial IgA deposits were present in $20.4 \%$ of donor biopsies in an ethnically diverse US population, $13.2 \%$ of living donors and $24.5 \%$ of deceased donor allografts, particularly from Hispanic or Asian donors [24]. In these patients, the IgA tended to clear over time and the 5 -year graft survival was similar to that in non-IgAcontaining allografts. It is, however, critical to know the original cause of kidney failure and also to have a donor biopsy with complete IF staining, in order to properly classify IgAN in the transplant as either de novo, recurrent, or donor related. The course of de novo IgAN generally depends on the expression of glomerular morphology, those with proliferative or crescentic lesions having a more rapid progression [1].

\section{Renal Pathology}

By light microscopy, the glomeruli can range from unaffected to mesangial hypercellularity with more severe cases demonstrating endocapillary hypercellularity, segmental glomerulosclerosis, and/or crescents. On IF, there are IgA-dominant deposits seen in the mesangium with co-localization of $\mathrm{C} 3$, kappa, and lambda and on occasion IgM or IgG of weaker intensity. On EM, mesangial or paramesangial electron-dense deposits are readily identified. An important distinction from IgA-dominant infection-related GN is the absence of subepithelial hump-like deposits and sometimes subendothelial deposits, in typical IgAN on the EM study.

\section{Infection-Related/Postinfectious Glomerulonephritis}

Definition and Background

Renal transplant recipients on immunosuppression are susceptible to a wide variety of infections (bacterial, viral, and fungal), for which routine posttransplant therapeutic prophylaxis is maintained [26]. The risk factors are diabetes mellitus, older age-group, females, intraoperative complications, and posttransplant immunosuppression. The sources of infection can vary from nosocomial, activation of latent infections, such as relapsed or oppor- 

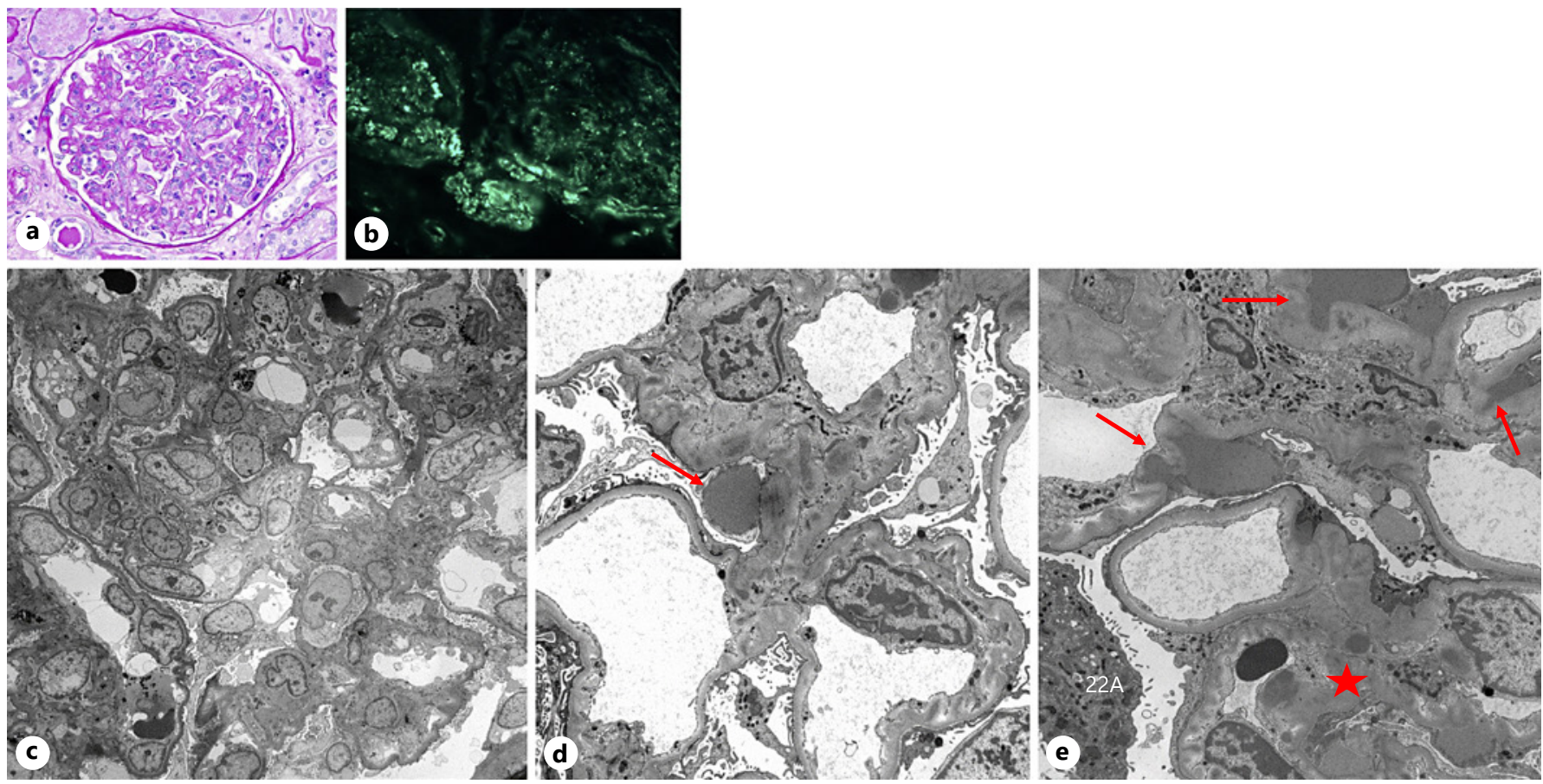

Fig. 4. De novo infection-related/postinfectious glomerulonephritis. a Glomerulus showing proliferative glomerulonephritis with endocapillary hypercellularity composed of mainly infiltrating inflammatory cells in a transplant patient with bacterial pneumonia (PAS, $\times 400)$. b Coarsely granular, scattered C3 glomerular deposits "starry sky pattern" are identified by IF along with strong stain- ing in the hilar arterioles $(C 3, \times 200)$. c-e EM images showing focal intraglomerular hypercellularity and scattered hump-like subepithelial electron dense deposits (arrows) including the basement membrane notches where they reflect on the mesangium, and focal mesangial deposits (asterisk). The foot processes are focally or partially effaced $(\times 1,800(\mathbf{c}), \times 6,000(\mathbf{d}, \mathbf{e}))$. tunistic forms or a community acquired infection, having a predilection within certain posttransplant periods [26]. The common bacterial pathogens encountered in renal transplant patients, regardless of the site of infection are Staphylococcus aureus, Enterocooci, Enterobacter, and Psuedomonas species, all capable of eliciting acute immune complex type of proliferative GN. Thus, an infection-related (concurrent to active infection) or a postinfectious immune complex glomerulonephritis (few weeks following the infection) may develop as a de novo disease within the allograft. This can be associated with bacteria, hepatitis $\mathrm{C}$, or hepatitis B viral infections and CMV infection $[10,25-27]$.

\section{Clinical Features}

The clinical presentation often includes acute renal failure, a nephritic syndrome with low complement levels, and rarely isolated hematuria and/or proteinuria, necessitating a transplant biopsy for a definitive diagnosis, particularly in those with subclinical or latent infections. Additional imaging, serologic, and other laboratory studies including blood and urine cultures for bacteria aid in pointing toward a more specific cause. Effective immunosuppression used to prevent a rejection process may prevent the ability of these patients to express the usual constitutional symptoms or clinical manifestations of a systemic or local infection. Although little data are available on the clinical impact, the prognosis appears relatively poor, with progressive deterioration of graft function, in severe cases.

\section{Renal Pathology}

Light microscopic findings are relatively heterogeneous $[25,27]$ and could range from minimal glomerular changes to focal or diffuse endocapillary proliferative and exudative changes (Fig. 4a). These can be accompanied by with or without cellular crescents or significant tubulointerstitial inflammation. The glomerular lesions may depend on the type and severity of the infection and host immune status. A range of different glomerular lesions associated with CMV infection have been documented in the literature, such as diffuse proliferative GN, IgA nephropathy, crescentic GN, and immunotactoid GN [27]. IF in all the reported cases reveals variable C3 

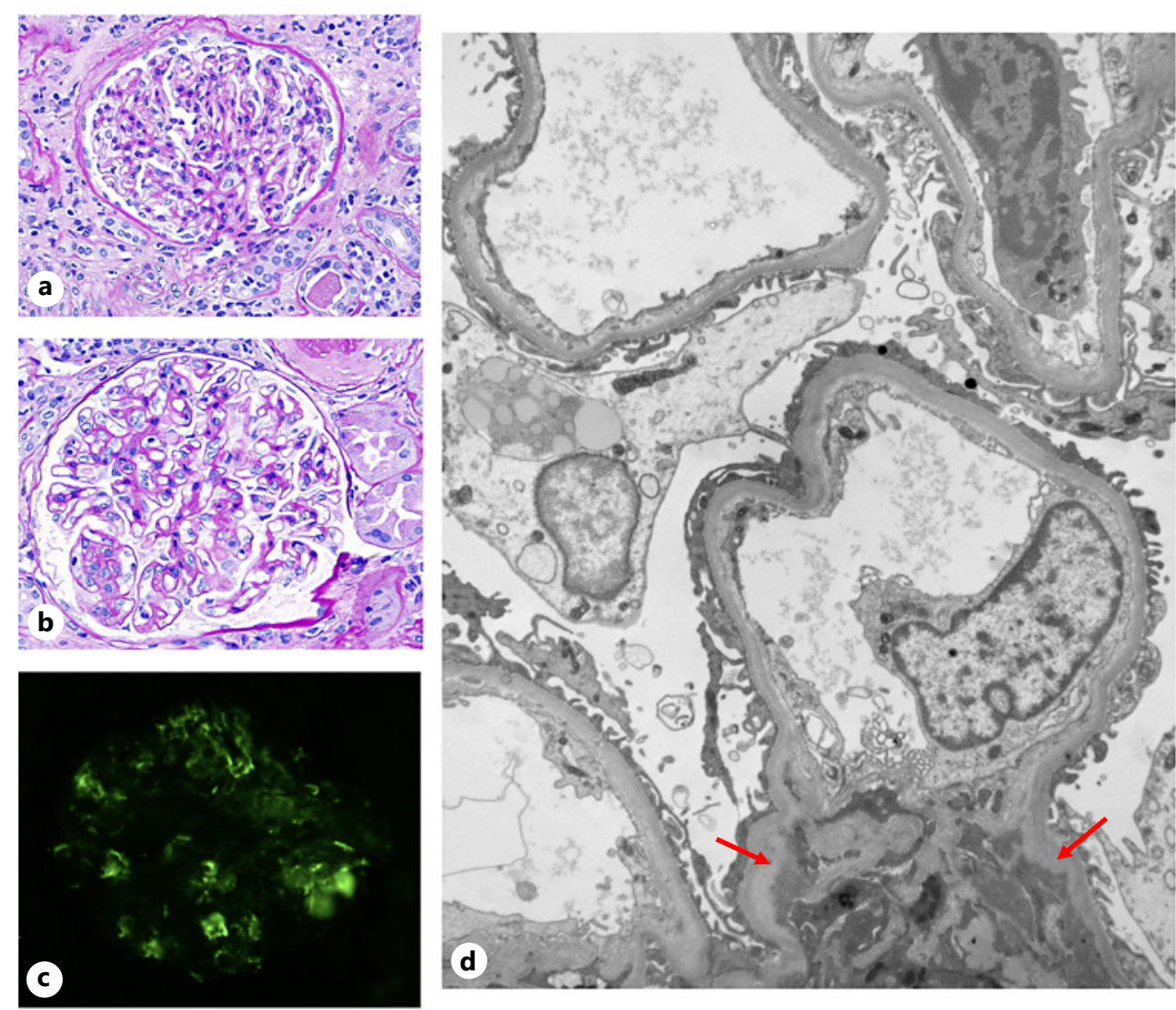
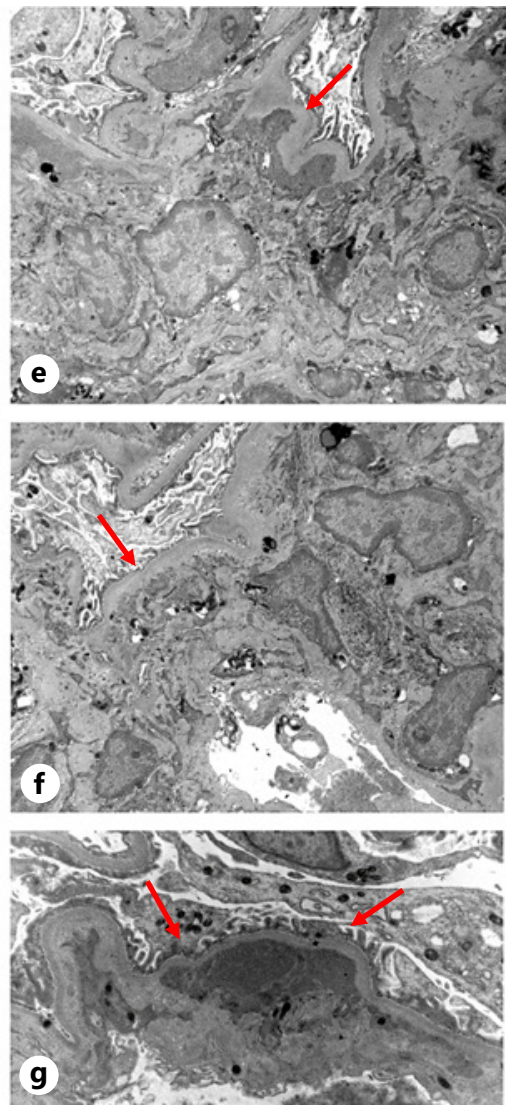

Fig. 5. De novo C1q nephropathy. a, b The glomeruli commonly display mild to moderate mesangial expansion containing increased cellularity, generally patent capillary lumina and normal thickness of the peripheral capillary walls (PAS $\times 400$ ). c Mainly granular deposits of dominant $\mathrm{Clq}$ are localized in the mesangium (C1q, $\times 200)$. $\mathbf{d}-\mathbf{g}$ EM images from glomeruli of 2 patients with $\mathrm{C} 1 \mathrm{q}$ nephropathy show finely granular deposits solely in the mesangial areas with accompanying hypercellularity. The glomerular capillary basement membranes are normal or mildly thickened without specific abnormalities. Despite subnephrotic or rarely nephrotic range proteinuria, the foot processes are often intact and epithelial cells may show mild swelling with protein resorption droplets $(\times 6,000(\mathbf{d}-\mathbf{f}), \times 10,000(\mathbf{g}))$. EM, electron microscopy deposits (Fig. 4b), with or without other immunoglobulin deposits (IgG, IgA, and IgM), as a scattered "starrysky" pattern, a rare case with C3 and IgA staining [25]. As expected, the characteristic "hump-like" subepithelial deposits or sometimes subendothelial and mesangial deposits are also observed by EM with focal to partial foot process effacement (Fig. 4c-e) [27]. This process can also superimpose on all forms of underlying diabetic kidney, in the renal allograft. While membranoproliferative pattern of GN develops with $\mathrm{HCV}$ infection with immune complex deposits [10], membranous type of GN is found with hepatitis B viral infection (personal experience). EM is useful to resolve the differences between HCV-associated MPGN and other similar de novo glomerular lesions which include transplant glomerulopathy and TMA.

De novo Diseases in Renal Transplantation

\section{Other De Novo Glomerular Diseases}

It is well known that almost any form of glomerular lesions can occur as de novo disease in the renal allograft, manifesting appropriate nephrotic or nephritic syndromes or renal dysfunction. So in this setting, a complete workup of the kidney biopsy including EM will aid in a definitive diagnosis. They include cases of steroid-sensitive minimal change nephrotic syndrome [28], anecdotal cases of membranoproliferative GN excluding HCV infection, C3 glomerulonephritis, fibrillary GN, proliferative glomerulonephritis with monoclonal IgG deposits, and AL amyloidosis [1,28-30]. In addition to donor-derived and recurrent diabetic kidney disease, de novo diabetic kidney disease is not uncommon in long-term grafts, usually preceded by several years of clinical posttransplant diabetes mellitus that may develop relatively rap- 
idly [31]. Typical diabetic parenchymal lesions including diabetic glomerulosclerosis are noted. A role for calcineurin inhibitors (CNIs) (cyclosporin and tacrolimus) and mTOR inhibitor (sirolimus) is suggested in promoting new-onset diabetes mellitus in transplant patients [1].

Larger systematic studies of allograft renal biopsies have also identified a subset of nonspecific immune complex glomerulopathies with no specific underlying etiologies, a significant number with concomitant rejection process $[32,33]$. These cases demonstrated varied composition of deposits, such as "full house," partial full house, IgA rich, C1q rich [32, 33], and mainly $\operatorname{IgM}[33]$. While it may raise the association of alloimmunity in some of these cases [32], the quality and quantity of such deposits assessed by routine IF and perhaps EM studies may provide further insights into such lesions.

De novo TMA, sharing similar pathologic features as in recurrent TMA, poses an important cause of graft dysfunction and failure, where the glomeruli and/or vasculature may be affected. However, recognizing the underlying etio-pathogenetic mechanisms, such as CNI toxicity, antibody-mediated rejection, CMV viral infection, or mutations involving the complement regulatory factors, is useful for further testing and management or a possible subsequent renal transplantation $[7,34,35]$.

\section{De Novo C1q Nephropathy}

Definition

C1q nephropathy is defined as the finding of glomerular mesangial staining and deposits of dominant or codominant C1q, in the absence of systemic lupus erythematosus. This is sometimes observed de novo in allografts, although the clinical significance of this finding is yet to be determined [36]. No known clinical association with rejection process, an infection, or other posttransplant treatment protocols exists. A few anecdotal case reports (in the setting of polyoma nephropathy) and 1 large retrospective study of 24 cases seen in both surveillance and for cause transplant biopsies are available. This is presumed to be a nonspecific binding of $\mathrm{Clq}$ to a polyanionic intrinsic or extrinsic antigen possibly related to an infection [36].

\section{Clinical Features}

Based on this report of 24 cases, no specific clinical profile is identified. Nearly $85 \%$ of the patients were Caucasian, $63 \%$ male, with an age range of $15-72$ years and over $70 \%$ were diagnosed 1 year or later posttransplantation. The usual clinical renal presentation was subnephrotic proteinuria (50\%) with rare cases of hematuria, while creatinine levels reflected other underlying disease processes in the al- lograft. Only $13 \%$ of the patients in this study were presensitized to donor-specific antibodies and 1 had antibodymediated rejection; thus, no significant association was made with any form of rejection process. Long-term followup has not shown a detrimental effect on graft survival [36].

\section{Renal Pathology}

The common LM lesions were mesangial hypercellularity (Fig. 5a, b) in $46 \%$ of the cases and FSGS in $21 \%$ and no abnormalities in $33 \%$. While it is not known how often routine IF with the full of antibody panel including $\mathrm{Clq}$ is performed on transplant kidney biopsies, a dominant or codominant $\mathrm{Clq}$ staining is necessary to make the diagnosis (Fig. 5b). Identification of glomerular mesangial electron-dense deposits by EM (Fig. 5c, d) observed in a majority of cases confirms the presence of $\mathrm{C} 1 \mathrm{q}$ deposits. No other glomerular capillary wall changes or alterations have been documented.

\section{Antiglomerular Basement Membrane-Mediated}

Glomerulonephritis in AS

Definition and Background

When anti-glomerular basement membrane-mediated glomerulonephritis (anti-GBM GN) occurs in a renal transplant biopsy, it is almost always attributed to male patients having a prior diagnosis of X-linked AS and a few females with autosomal-recessive AS, who progressed to ESKD. This is a result of de novo alloantibody response by the recipient mainly against the alpha 5 chain in the noncollagenous portion of GBM type IV collagen in the donor kidney, although this may not be detected in routine serologic studies. A few patients, usually autosomal-recessive AS type, may have antibodies cross-reacting with alpha 3 and/or alpha 4 chains of type IV collagen. This is in contrast to the autoantibodies primarily to alpha 3 chains, in anti-GBM disease involving the native kidneys. The degree of the antibody response by the recipient depends on the type and severity of the underlying genetic aberration. A truncating mutation, such as a deletion or nonsense mutations of alpha 5 chain, has been implicated in early onset of $\operatorname{ESKD}[37,38]$ in AS patients. It may also develop in successive transplants in the same patient.

\section{Clinical Features}

The humoral response to produce anti-GBM antibodies in known patients with end stage secondary to AS may be detected within a few days to several weeks or months after transplantation. Although circulating anti-GBM antibodies are detected in $10-60 \%$ of the AS patients posttransplantation [38], only about $3-5 \%$ of these seroposi- 
tive cases develop actual morphological features of antiGBM GN [38]. These present with an acute nephritic syndrome or acute renal failure, hematuria, and red blood cell casts, which necessitate a transplant renal biopsy for confirmation of the diagnosis.

\section{Renal Pathology}

The light and IF findings resemble those observed in native kidney disease. The glomeruli display fibrinoid-necrotizing lesions with cellular crescents involving $50-80 \%$ of the glomeruli without any evidence of intraglomerular cellularity or deposits. This is accompanied by active tubulointerstitial inflammation and edema [7]. The typical IF finding of diffuse linear IgG staining along the glomerular basement membrane is observed with co-localization of C3. As in the native kidney disease, no specific electron microscopic alterations or deposits are identified. De novo anti-GBM GN should be differentiated from other de novo or recurrent diseases, such as pauci-immune (associated with antineutrophil cytoplasmic antibodies) crescentic GN (IF negative) or immune complex-mediated GN (granular IF staining), sometimes encountered in infection-related, autoimmune, or paraprotein-associated diseases. These patients are managed as other forms of crescentic GN, seen in native kidney disease. A positive clinical history of previous AS is helpful.

\section{Recurrent Proteinuria in Congenital Nephrotic \\ Syndrome}

Definition and Pathological Findings

Congenital nephrotic syndrome of the Finnish type in infancy during the first 3 months of life is caused by autosomal recessive mutations of the gene "nephrin," a podocyte slit-diaphragm-associated protein. This is particularly seen in those with a homozygous genotype Fin-major/ Fin-major, which results in complete lack of nephrin. The significant recurrent proteinuria and its complications lead to renal failure or necessitate therapeutic nephrectomy, requiring renal transplantation. A small proportion of these cases with ESKD may have a risk of developing recurrent posttransplant nephrotic syndrome in $24-35 \%$ of cases, attributed to de novo alloantibody response against nephrin in the donor kidney, with circulating anti-nephrin antibodies [39]. By light microscopy, the glomeruli may not show any other changes or mesangial hypercellularity. By EM, normal-appearing glomerular basement membranes, with endothelial swelling and/or widespread podocyte injury leading to foot process effacement are seen $[7,39]$. Plasmapheresis, immunosuppressive therapy, and anti-CD20 antibody have been helpful [39].

\section{Conclusions}

De novo renal diseases in transplantation together with recurrent diseases account for the 3rd highest cause of allograft dysfunction and graft loss based on large studies and transplant registries. Virtually, any form of renal lesion can occur as part of de novo diseases. However, certain special categories of renal lesions with unique features are encountered as de novo diseases in the transplant setting that deserves attention and have been reviewed in this article. For a proper diagnosis of de novo diseases in renal transplants, knowledge of prior kidney disease before endstage, routine clinical urine testing for proteinuria and hematuria, and appropriate serologic testing are essential. In this regard, a transplant kidney biopsy subjected to light, IF, and EM is deemed as the gold standard. Often, the histologic or ultrastructural findings may be modified as a result of ongoing immunosuppressive therapy, and other inherent donor and recipient characteristics. The most common forms of de novo diseases are membranous glomerulonephritis, focal segmental sclerosis, IgA nephropathy, infection-related renal lesions, and rarely thrombotic microangiopathy [1], most importantly complement defect-associated hemolytic-uremic syndrome with significant impact on graft function and graft loss. EM aids in early identification, diagnosis, and staging of the lesions.

\section{Acknowledgement}

The authors would like to express their gratitude to the EM technical staff (Mr. Michael Ganger, Mrs. Lilian Antonio, and Ms. Melanie Wilson) for providing EM images and Mr. Mikhail Snigirev for secretarial and digital assistance in the preparation of this manuscript.

\section{Conflict of Interest Statement}

The authors have no conflicts of interest to declare.

\section{Funding Sources}

No funding resources were used for this work.

\section{Author Contributions}

Surya V. Seshan and Steven P. Salvatore participated in preparation of manuscript and assembling images and references. 


\section{References}

1 Ponticelli C, Moroni G, Glassock RJ. De novo glomerular diseases after renal transplantation. Clin J Am Soc Nephrol. 2014;9(8):147987.

2 Lim WH, Shingde M, Wong G. Recurrent and de novo glomerulonephritis after kidney transplantation. Front Immunol. 2019;10: 1944.

3 Seshan SV. De novo renal disease. In: Cagle PT, Yerian L, Truong LD, editors. Atlas of transplant pathology. Illinois: CAP Press; 2015. p. 123-29.

4 Amer H, Fidler ME, Myslak M, Morales P, Kremers WK, Larson TS, et al. Proteinuria after kidney transplantation, relationship to allograft histology and survival. Am J Transplant. 2007;7(12):2748-56.

5 Wavamunno MD, O'Connell PJ, Vitalone M, Fung CL, Allen RD, Chapman JR, et al. Transplant glomerulopathy: ultrastructural abnormalities occur early in longitudinal analysis of protocol biopsies. Am J Transplant. 2007; 7(12):2757-68

6 Royal V, Zee J, Liu Q, Avila-Casado C, Smith AR, Liu G, et al. Ultrastructural characterization of proteinuric patients predicts clinical outcomes. J Am Soc Nephrol. 2020;31(4): 841-54.

7 Nickeleit V, Mengel M, Colvin RB. Renal transplant pathology: de novo and recurrent renal diseases. In: Jennette JC, D'Agati VD, Olson JL, editors. Silva FG heptinstall's pathology of the kidney. 7th ed. Philadelphia, PA: LWW; 2015. p. 1415-24.

8 Batal I, Vasilescu ER, Dadhania DM, Adel AA, Husain SA, Avasare R, et al. Association of HLA typing and alloimmunity with posttransplantation membranous nephropathy: a multicenter case series. Am J Kidney Dis. 2020;76(3):374-83.

9 Larsen CP, Walker PD. Phospholipase A2 receptor (PLA2R) staining is useful in the determination of de novo versus recurrent membranous glomerulopathy. Transplantation. 2013;95(10):1259-62.

10 Cruzado JM, Carrera M, Torras J, Grinyó JM. Hepatitis $C$ virus infection and de novo glomerular lesions in renal allografts. Am J Transplant. 2001;1:171-8.

11 Sethi S. New “antigens” in membranous nephropathy. J Am Soc Nephrol. 2021;32(2): 268-78.

12 Ronco P, Plaisier E, Debiec H. Advances in membranous nephropathy. J Clin Med. 2021; 10(4):607-25.

13 Wang G, Sun L, Dong H, Wang Y, Xu X, Zhao $Z$, et al. Neural epidermal growth factor-like 1 protein-positive membranous nephropathy in Chinese patients. Clin J Am Soc Nephrol. 2021;16(5):727-35. Published online ahead of print, 2021 Apr 13.
14 Rodriguez EF, Cosio FG, Nasr SH, Sethi S, Fidler ME, Stegall MD, et al. The pathology and clinical features of early recurrent membranous glomerulonephritis. Am J Transplant. 2012;12(4):1029-38.

15 Ehrenreich T, Churg J. Pathology of membranous nephropathy, 1968. In: Sommers SC, editor. Kidney pathology decennial, 19661975. New York: Appleton-Century-Crofts; 1975. p. 389-433.

16 Santoriello D, Husain SA, De Serres SA, Bomback AS, Crew RJ, Vasilescu ER, et al. Donor APOL1 high-risk genotypes are associated with increased risk and inferior prognosis of de novo collapsing glomerulopathy in renal allografts. Kidney Int. 2018;94(6):1189-98.

17 Nadasdy T, Allen C, Zand MS. Zonal distribution of glomerular collapse in renal allografts: possible role of vascular changes. Hum Pathol. 2002;33(4):437-41.

18 Hayes JM, Steinmuller DR, Streem SB, Novick AC. The development of proteinuria and focal-segmental glomerulosclerosis in recipients of pediatric donor kidneys. Transplantation. 1991;52(5):813-7.

19 Jiang Z, Liang Y, Zhong T, Yang S, Chen Y, Huang $G$, et al. Pediatric donor glomerulopathy is a possible cause of abnormal urinalysis in adults receiving small pediatric donor kidneys. Transplantation. 2020;104(8): 1695702.

20 Bohlin AB, Berg U. Renal functional adaptation of the adult kidney following transplantation to the child. Kidney Int. 1991;39(1): 129-34.

21 Muramatsu M, Hamasaki Y, Mizutani T, Takahashi Y, Hashimoto J, Kubota M, et al. Comparison of live donor predonation and post-transplant kidney volumes and glomerular size in pediatric patients weighing less than $15 \mathrm{~kg}$ : a retrospective study. Transpl Int. 2020;33(8):878-86

22 Tang Z, Ji SM, Chen DR, Wen JQ, Chen JS, Liu $\mathrm{ZH}$, et al. Recurrent or de novo IgA nephropathy with crescent formation after renal transplantation. Ren Fail. 2008;30(6):611-6.

23 Cazorla-López JM, Wu J, Villanego-Fernández F, Naranjo-Muñoz J, Vigara-Sánchez LA, García-García-Doncel A, et al. IgA nephropathy after renal transplant: recurrences and De novo cases. Transplant Proc. 2020;52(2): 515-8.

24 Gaber LW, Khan FN, Graviss EA, Nguyen DT, Moore LW, Truong LD, et al. Prevalence, characteristics, and outcomes of incidental IgA glomerular deposits in donor kidneys. Kidney Int Rep. 2020;5(11):1914-24.

25 Moroni G, Papaccioli D, Banfi G, Tarantino A, Ponticelli C. Acute post-bacterial glomerulonephritis in renal transplant patients: description of three cases and review of the literature. Am J Transplant. 2004;4(1):132-6.

26 Karuthu S, Blumberg EA. Common infections in kidney transplant recipients. Clin J Am Soc Nephrol. 2012;7(12):2058-70.
27 Plumb TJ, Greenberg A, Smith SR, Butterly DW, Pham TT, Fields TA, et al. Postinfectious glomerulonephritis in renal allograft recipients. Transplantation. 2006;82(9):1224-8.

28 Java A, Gaut JP, Brennan DC. De novo membranoproliferative glomerulonephritis type III in a renal transplant patient: case report and review of literature. Transpl Int. 2012;25: e56-61.

29 Albawardi A, Satoskar A, Von Visger J, Brodsky S, Nadasdy G, Nadasdy T. Proliferative glomerulonephritis with monoclonal IgG deposits recurs or may develop de novo in kidney allografts. Am J Kidney Dis. 2011;58(2): 276-81.

30 Qian Q, Nasr SH, Fidler ME, Cornell LD, Sethi S. De novo AL amyloidosis in the kidney allograft. Am J Transplant. 2011;11(3):60612.

31 Bhalla V, Nast CC, Stollenwerk N, Tran S, Barba L, Kamil ES, et al. Recurrent and de novo diabetic nephropathy in renal allografts. Transplantation. 2003;75(1):66-71.

32 Chin KK, Charu V, O'Shaughnessy MM, Troxell ML, Cheng XS. Histologic case definition of an atypical glomerular immune-complex deposition following kidney transplantation. Kidney Int Rep. 2020;5(5):632-42.

33 Gough J, Yilmaz A, Yilmaz S, Benediktsson H. Recurrent and de novo glomerular immunecomplex deposits in renal transplant biopsies. Arch Pathol Lab Med. 2005;129(2):231-3.

34 Satoskar AA, Pelletier R, Adams P, Nadasdy GM, Brodsky S, Pesavento T, et al. De novo thrombotic microangiopathy in renal allograft biopsies-role of antibody-mediated rejection. Am J Transplant. 2010;10(8):180411.

35 Le Quintrec M, Lionet A, Kamar N, Karras A, Barbier S, Buchler M, et al. Complement mutation-associated de novo thrombotic microangiopathy following kidney transplantation. Am J Transplant. 2008;8(8):1694-701.

36 Said SM, Cornell LD, Valeri AM, Sethi S, Fidler ME, Cosio FG, et al. C1q deposition in the renal allograft: a report of 24 cases. Mod Pathol. 2010;23(8):1080-8.

37 Kashtan CE. Renal transplantation in patients with Alport's syndrome: patient selection, outcomes, and donor evaluation. Int J Nephrol Renovasc Dis. 2018;11:267-70.

38 Querin S, Noel LH, Grunfeld JP, Droz D, Mahieu P, Berger J, et al. Linear glomerular IgG fixation in renal allografts: incidence and significance in Alport's syndrome. Clin Nephrol. 1986;25(3):134-40.

39 Holmberg C, Jalanko H. Congenital nephrotic syndrome and recurrence of proteinuria after renal transplantation. Pediatr Nephrol. 2014;29(12):2309-17. 